\title{
Scritti sparsi di Ignazio Silone
}

\author{
Vincenza A. C. Tudini
}

Malgrado le sue lacune, l'elenco di scritti che segue dovrebbe rendere più accessibile l'opera sparsa di Silone ed offrire nuovi spunti a chiunque s'interessi di tale autore o del contesto storico in cui egli scrisse. Gli scopi della bibliografia sono principalmente di carattere enumerativo in quanto essa costituisce una versione non critica e più dettagliata della guida agli scritti sparsi di Silone contenuta nel volume di Luce D'Eramo (503-57). Tale guida, senza cui l'elenco che segue sarebbe stato pressoché impossibile, è infatti indispensabile per chi desideri approfondire il rapporto dell'attività giornalistica con l'evoluzione politica e letteraria di Silone. Tra l'altro, il volume di D'Eramo pare sia stato scritto con la collaborazione dello scrittore stesso.

La presente bibliografia porta a conoscenza molti titoli specifici e talvolta sconosciuti di pubblicazioni saggistiche e giornalistiche siloniane. Malgrado la possibilità di continuare a corredarlo di altri titoli di Silone, l'elenco di scritti sparsi è più completo delle bibliografie attualmente disponibili. Servendo come punto di partenza, esso mette in rilievo la necessità della pubblicazione in volume degli scritti più significativi di Silone. Il compito di un eventuale biografo di Silone potrebbe essere facilitato dall'uso dell'elenco. Inoltre, per gli studiosi di Silone che non abitano in Italia o in Svizzera, l'elenco che segue rende possibile l'accesso, tramite un servizio di prestito interbibliotecario, a titoli finora inaccessibili o sconosciuti. Le biblioteche principali presso cui sono reperibili gli scritti elencati sono la Biblioteca Nazionale di Roma, la Biblioteca Comunale di Milano, l'Istituto Feltrinelli di Milano e la Biblioteca Statale di Zurigo.

Anche senza una valutazione critica degli articoli elencati, è possibile desumere (dal titolo e dalla rivista in cui sono stati pubblicati) un'indicazione delle questioni che hanno interessato Silone. Ciò costituisce un passo avanti rispetto a quanto disponibile attualmente.

L'attività giornalistica è un aspetto rilevante della vita di Silone, e serve ad illuminare non solo il suo impegno politico, ma anche la sua attività letteraria. Benché verso la fine degli anni ' 40 egli abbandonasse la politica di partito, essendo precedentemente passato dal comunismo al socialismo per poi continuare il suo impegno senza affiliazioni partitiche, da 'cristiano senza chiesa e socialista senza partito', egli continuò a farsi sentire, non solo nell'attività letteraria, ma anche attraverso l'Associazione per la Libertà della Cultura, di cui fondò e diresse la sezione italiana nel 1950. Egli si servì prevalentemente di riviste neutrali come Tempo Presente, di cui fu direttore assieme a Nicola Chiaromonte, finché la rivista fu costretta a chiudere per mancanza di fondi 
nel dicembre del 1968. Durante gli anni '50 e '60 furono numerosi gli interventi su questioni importanti dell'epoca, non solo tramite Tempo Presente, ma anche con altre pubblicazioni giornalistiche, come è evidente dall'elenco dal numero 71 al 172. A giudicare dal numero di articoli scritti durante il ventennio $1950-70$, rispetto agli anni di militanza che lo precedono, pare che per Silone l'espressione giornalistica fosse più che mai una necessità che colmava la mancanza di espressione attraverso un'organizzazione politica.

Gli scritti siloniani sono elencati in ordine cronologico. Non includo interviste o articoli poi pubblicati in volumi più noti come Uscita di sicurezza o Memoriale dal carcere svizzero, i quali comunque riuniscono solo una piccola parte della saggistica siloniana. I numeri 23, 26, 31 e 176 dell'elenco sono stati aggiunti gentilmente da Brian Moloney dell'Università di Wollongong.

Le mie ricerche riguardo ai famosi tre articoli che Secondino Tranquilli diciassettenne inviò all'Avanti! sono state infruttuose. Tali articoli, segnalati da Silone (Uscita 76-79), denunciano la corruzione di coloro che erano incaricati della ricostruzione edilizia del suo paese dopo il terremoto del 1915.

Non è stato nemmeno possibile reperire, seguendo le indicazioni di D'Eramo (503), gli articoli apparsi per la prima volta nel 1923 sul settimanale di Barcellona La Batalla. L'elenco di articoli apparsi su L'avvenire dei lavoratori di Zurigo, pubblicato tra il febbraio e l'ottobre del 1944 e diretto da Silone, non è completo data la difficoltà di attribuirgli articoli anonimi. Elisa Signori offre spunti utili in proposito:

Gli articoli sono anonimi o siglati con pscudonimi, ad eccezione dei testi tradotti da libri o da riviste stranicre o dei pezzi di autore svizzcro, tuttavia c̀ abbastanza agevole riconoscere la mano di Silone nello stile inconfondibile di molti editoriali, anche perché egli ne riutilizzò qualche brano in sue successive esperienze pubblicistiche, particolarmente in Europa socialista. Ci pare, ad csempio, incontestabile l'attribuzione a Silone di una originale rubrica dal titolo Vocabolario, che, specie nei primi numeri, propone spregiudicate riflessioni su alcuni termini chiave del linguaggio politico: massa, idcologia, nazionale, patriotta, razza, radicale, unitario, ecc. Ne risulta un ironico repertorio dei tic linguistici propri ai diversi gruppi politici, dai democratici ai comunisti compresi. (114)

Bisogna inoltre prender nota del fatto che Silone ha praticamente ripudiato l'opera del suo periodo di militanza nel P.C.I.:

Per il decennio che va dal 1917, quando Silone si fece rivoluzionario, fino al 1927 in cui riparò per la prima volta in Svizzera, . . . lo scrittore non si sente di menzionare nessun articolo di giornale di cui ora possa rivendicarc la paternità". (D’Eramo 504)

Scritti di tale periodo che non sono menzionati nel seguente elenco sono reperibili presso l'archivio del P.C.I. e l'Archivio Gramsci a Roma. Le ragioni dell'atteggiamento dello scrittore verso i suoi scritti giovanili sono piuttosto complesse e abbastanza evidenti a chi abbia letto i suoi romanzi. Come Pietro Spina de Il seme sotto la neve, piuttosto che Pietro Spina ancora militante 
di Vino e pane, Silone è consapevole delle inadeguatezze della politica di partito, e si trova a disagio con la sua vita di giovane militante comunista. Giudicandosi retrospettivamente, Silone osserva con maggiore chiarezza ed esperienza le sue illusioni utopiche dei tempi in cui era una figura chiave del movimento comunista, di cui in seguito vide il volto repressivo e imperialista che gli causò il distacco dal P.C.I. e il "lutto della gioventù" (Uscita 116).

\section{Elenco degli scritti sparsi di I. Silone}

1 "Intervento all'VIII congresso della Federazione giovanile socialista italiana del 27 gennaio 1921”. L'avanguardia. 15.6-7 (febbraio 1921).

2 “Concentrazione repubblicana". L'Unità (2 settembre 1926).

3 "Elementi per uno studio del P.N.F. (Borghesia, piccola borghesia e fascismo)”. Stato operaio 1.8 (agosto 1927). [Firmato S. Tranquilli.]

4 "Borghesia, piccola borghesia e fascismo". Stato operaio 2.4 (aprile 1928). [Firmato S. Tranquilli.]

5 "Sviluppo e funzioni del sindacalismo fascista". Stato operaio 2.10 (novembre-dicembre 1928). [Firmato S. Tranquilli.]

6 "La situazione italiana alla vigilia del plebiscito". Stato operaio 3.2 (febbraio 1929). [Firmato S. Tranquilli.] Rpt. "La società italiana e il fascismo". Tempo Presente 7.12 (dicembre 1962).

7 "Il congresso internazionale antifascista". Stato operaio 3.3 (marzo 1929). [Firmato S. Tranquilli.]

8 "Riformismo e fascismo". Stato operaio 4.3 (marzo 1930). [Firmato con lo pseudonimo "Pasquini".]

9 "Dichiarazione di voto al Comitato centrale del Partito comunista italiano". Stato operaio 4.4 (aprile 1930).

10 "Gugliemo Ferrero". Stato operaio 4.5-6 (maggio-giugno 1930).

11 "Un caso di malavita politica: lettere di Pasquini all'Ufficio politico del Partito comunista svizzero". Stato operaio 5.5 (maggio 1931).

12 "Alfredo Rocco, der Schopfer der juristischen Doktrin des Fascismus". Information 1 (giugno 1932).

13 "Krise, Krieg und Abrustung”. Information 2 (luglio 1932).

14 "Was ist Fascismus? Versuch einer definition". Information 3 (agosto-settembre 1932).

15 "Katholizismus und Fascismus: die Katholische Kirche als Agrarstaat". Information 4 (ottobre 1932).

16 "Stadt und Land: Katholizismus und Fascismus". Information 5 (novembre 1932).

17 "Der Christus von Kazan". Information 6 (dicembre 1932). Die Weltwoche Zurich (1 maggio 1970).

18 "Die professionellen Verbrecher und der Schutz des Privateigentums". Information 8 (marzo 1933).

19 "Die erste Phase einer fascistischen Diktatur; Partei und Regierung". Information 9 (aprile 1933).

20 "Die Korporationen in Italien". Information 6 (febbraio 1934). 
21 Der Fascismus; seine Entstehung und seine Entwicklung. Trad. tedesca di Gritta Baerlocher. Zurich: Europa Verlag, 1934. Frankfurt a. M.: Verlag Neue Kritik, 1971.

22 "Una lettera a Mosca". International Review (novembre 1936).

23 "Rhetoric of life". The Nation (24 aprile 1937).

24 "Lettera aperta di Silone: 5 settembre 1937". Littérature engagée. Ed. Andrè Gide. Parigi: Gallimard, 1950: 187-90.

25 "Nuovo incontro con Giuseppe Mazzini". [1938]. Il Ponte (gennaio 1949). Rpt. a cura dell'Associazione mazziniana italiana: 1978.

26 Introduzione a The Living Thoughts of Mazzini. Londra: Cassel, 1939: $1-32$.

27 "La fine d'un concordato". [1940]. Il verdetto di tre decenni. Ed. Julien Steinberg. Milano: 1952: 470-75.

28 "Ferrero and the Decline of Civilizations". Partisan Review 9.5 (settembreottobre 1942). "Guglielmo Ferrero e il tramonto delle civiltà". Comunità Ivrea (luglio-agosto 1949).

29 "The Things I stand for". The New Republic (2 novembre 1942).

30 "A Call for Civil Disobedience-Manifesto of the Italian Socialist Party". Socialist Commentary (dicembre 1942).

31 "Ad occhi aperti". L'avvenire dei lavoratori (1 febbrario 1944).

32 "Sulla letteratura moderna". L'avvenire dei lavoratori 9 (s. a.).

33 "Due posizioni false: l'anticomunismo fazioso e la capitolazione di fronte al partito comunista". L'avvenire dei lavoratori (1 marzo 1944).

34 "I socialisti e la massa". L'avvenire dei lavoratori (15 marzo 1944).

35 "Il problema della federazione europea". L'avvenire dei lavoratori (15 marzo 1944).

36 "Libertà di pensiero e disciplina politica". L'avvenire dei lavoratori (15 aprile 1944).

37 "Discussione sul socialismo". L'avvenire dei lavoratori (15 aprile 1944).

38 "Il carattere della rivoluzione italiana". L'avvenire dei lavoratori (15 aprile 1944).

39 "Progetto americano di Community of States". L'avvenire dei lavoratori (15 giugno 1944).

40 "Il pensiero federativo di Carlo Cattaneo". L'avvenire dei lavoratori (15 agosto 1944).

41 “L'Italia in periodo di transizione”. Avanti! (15 agosto 1944).

42 "I problemi europei". Avanti! (21 ottobre 1944).

43 "Silone's message to Italy". Socialist Commentary (novembre 1944).

44 "Alcuni dati del problema politico italiano". Avanti! (12 dicembre 1944).

45 "Da Paneuropa alla Federal Union". Libera Stampa, giornale socialista del Canton Ticino (2 gennaio 1945).

46 "I socialisti, la guerra e la pace". Socialismo Roma (12 marzo 1945).

47 "Ideologie e politica". Mercurio Roma (febbraio 1945). Rpt. Europa Socialista Roma (20 aprile 1947). 
48 "L'esigenza di un pacifico ordinamento democratico non può risolversi soltanto con mezzi giuridici". La Città Libera (26 aprile 1945).

49 "Solo la verità ci potrà salvare". Avanti! (23 maggio 1945). Rpt. in parte Tempo Presente (gennaio 1967).

50 "Pro-memoria". Socialismo (giugno-luglio 1945).

51 "Punti fondamentali". Critica Sociale (15 settembre 1945).

52 "Superare l'anti-fascismo". Avanti! (27 ottobre 1945).

53 “L'Internazionale Socialista”. Europa Socialista (16 marzo 1946).

54 "Autocritica". Avanti! 14 luglio 1946.

55 Intervento al convegno "L'unità e la pace nel mondo". Roma (ottobre 1947). Europa federata. Milano: Edizioni di Comunità, 1947.

56 "Partito in formazione". Europa Socialista (11 gennaio 1947).

57 “Come riorganizzare il movimento socialista?” Europa Socialista (23 febbraio 1947).

58 "La crise du socialisme italien". Revue Socialiste Parigi (marzo 1947).

59 “Punte di trasformismo nel P.S.L.I.". Europa Socialista (2 marzo 1947).

60 "Di nuovo una 'Carta". Europa Socialista (9 marzo 1947).

61 “Per la difesa del vecchio P.S.I.". Critica Sociale (9 marzo 1947). [Lettera di M. Gina a Silone con risposta di Silone.]

62 "Le condizioni della rinascita". Europa Socialista (13 aprile 1947).

63 "Perché la politica deve emanciparsi dalle ideologie". Europa Socialista (20 aprile 1947).

64 “Lelio Basso esalta la scissione”. Europa Socialista (27 aprile 1947).

65 “Nenni per l'unità socialista?” Europa Socialista (1 giugno 1947).

66 "Sulla dignità dell'intelligenza: e l'indegnità degli intellettuali". La Fiera Letteraria (3 luglio 1947). Rpt. "On the Place of the Intellect and the Pretensions of the Intellectual". The Intellectuals. Ed. George B. Huszan. New York: Free Press of Glencoe, 1947: 261-66. Rpt. Horizon (dicembre 1947).

67 "L’Abruzzo". Attraverso l'Italia: Abruzzo e Molise. Vol. 14. Milano: Touring Club Italiano, 1948: 8-13.

68 “Les vaincus invincibles". Révolution Proletarienne Parigi (maggio 1949).

69 "La società e il nostro destino". Il Ponte (ottobre 1949). [Dal discorso inaugurale del P.E.N. Club (Venezia, 11 settembre 1949).]

70 "Ricordo di Angelo Tasca". Prefazione a Angelo Tasca. Nascita e avvento del fascismo. L'Italia dal 1918 al 1922. Firenze: La Nuova Italia, 1950. Rpt. 1963: ix-xix.

71 "La libertà di sognare". La Fiera Letteraria (30 luglio 1950).

72 "Nel bagaglio dell'esule". Esperienze e studi socialisti. A cura di Critica Sociale. Firenze: La Nuova Italia, 1954: 301-15.

73 "Un'opinione sul romanzo". La Fiera Letteraria (11 aprile 1954).

74 “Terra di santi e di muratori”. La Fiera Letteraria (11 aprile 1954).

75 "La narrativa e il sottosuolo meridionale". Quaderni di prospettive meridionali 1 (gennaio 1956). Rpt. Italian Quarterly 1-2 (1957). 
76 "Uno scrittore socialista". La Giustizia (23-24 febbraio 1956).

77 "Primo incontro con Dostoievsky". La Fiera Letteraria 11.10 (4 marzo 1956).

78 "Il lievito del cuore". Prospettive meridionali (marzo 1956). Rpt. La Giustizia (2-3 aprile 1956). [Soggetto cinematografico.]

79 "Ideologia e realtà sociale". Tempo Presente 1.1 (aprile 1956).

80 "Risposte di Ignazio Silone a '9 domande sullo stalinismo". Nuovi argomenti (maggio-giugno 1956): 106-9. Rpt. Preuves Parigi (luglio 1956).

81 "Agenda". Tempo Presente 1.3 (giugno 1956).

82 "Agenda: Fantascienza. Rispetto per gli anarchici. Quali prospettive". Tempo Presente 1.5 (agosto 1956).

83 "Lo scrittore e lo stato". La Giustizia (29-30 agosto 1956). Rpt. Comprendre Parigi (settembre 1956).

84 "Agenda: Dignità dell'intelligenza". Tempo Presente 1.8 (novembre 1956).

85 "Invito ad un esame di coscienza". Tempo Presente 1.9 (dicembre 1956).

86 "Un dialogo difficile: dal disgelo al neo-stalinismo". Tempo Presente 2.2 (febbraio 1957). Rpt. "A Troubled Dialogue". Encounter (giugno 1957).

87 "Dialogo impossibile". Tempo Presente 2.4 (aprile 1957).

88 "Letteratura e politica". Critica Sociale (20 aprile 1957).

89 "Agenda: Gli apparati e la democrazia". Tempo Presente 2.5 (maggio 1957). Rpt. Critica Sociale (5 giugno 1957).

90 "Agenda: Il Barodet". Tempo Presente 2.6 (giugno 1957).

91 "Gli apparati". Volontà Napoli (30 giugno 1957). [Lettera di Silone a Giovanna Berneri, con risposta di Berneri.]

92 "Agenda: Autocritica laicista. Conferenzieri. Un apparato in azione. Popoff. Alla ricerca del tempo perduto". Tempo Presente 2.8 (agosto 1957).

93 "Agenda: Il professor Dossetti in famiglia. Teologia e amministrazione. Un crocifisso". Tempo Presente 2.9-10 (settembre-ottobre 1957).

94 "Les Soviets au pouvoir". Demain (5 novembre 1957).

95 "Il socialismo della povera gente". Corrispondenza Socialista (novembre 1957).

96 “Agenda: Thomas Mann e il dovere civile. Pensatore politico?" Tempo Presente 3.1 (gennaio 1958).

97 "Agenda". Tempo Presente 3.2 (febbraio 1958).

98 "Rettifiche e conferme su Thomas Mann". Tempo Presente 3.3 (marzo 1958).

99 "L'ideologia come diversivo". Tempo Presente 3.6 (giugno 1958).

100 "Agenda: Un pugno contro un paralitico. Messa a punto demistificatoria. La lezione di Pierre Monatte”. Tempo Presente 3.7 (luglio 1958).

101 "Agenda: Giustificazioni errate. L'integrazione impossibile". Tempo Presente 3.8 (agosto 1958).

102 "Apparati di partito e democrazia". Tempo Presente 3.11 (novembre 1958).

103 "Agenda: Cronache della steppa. Gare meschine. Una sezione socialista. Contro corrente". Tempo Presente 4.3 (marzo 1959). 
104 "Agenda: Sui congressi d'intellettuali. Scrittori e artisti negri a Roma. Divide et impera. Politique d'abord. Nazioni da aggiornare". Tempo Presente 4.4 (aprile 1959).

105 "L'unità politica dei cattolici italiani e il controllo della S. Sede sulla D.C.”. Tempi Moderni Roma (giugno 1959).

106 “Agenda: Berlino". Tempo Presente 4.8 (agosto 1959).

107 "Con chi dialogare". Tempo Presente 4.9-10 (settembre-ottobre 1959).

108 “Agenda: Malattie croniche”. Tempo Presente 4.11 (novembre 1959).

109 "L'arte del dialogo". Tempo Presente 4.12 (dicembre 1959).

110 "I periodici di cultura". Stampa d'oggi. Firenze: Vallecchi, 1959: 61-78.

111 Introduzione a AA. VV. A trent'anni dal Concordato. Firenze: 1959: 11-19.

112 "La parola scritta di Corrado Alvaro". La Giustizia (27 marzo 1960).

113 "Agenda: Democrazia cifrata. Relazioni culturali con la Russia". Tempo Presente 5.4 (aprile 1960).

114 "Il segreto dello scrittore". La Giustizia (1 maggio 1960).

115 "Agenda: La nuova utopia. La negazione della negazione. Il cane e il coniglio elettrico. La minestra di Stato". Tempo Presente 5.12 (dicembre 1960).

116 "Le nuove edizioni di Capolago e gli anni della guerra". Egidio Reale e il suo tempo. Firenze: La Nuova Italia, 1960: 151-70. Rpt. "Le edizioni di Capolago". Introduzione a La mostra della Tipografia Elvetica di Capolago. Catalogo. 1960.

117 "La condizione dell'uomo nella prospettiva del benessere". Le conferenze dell'Associazione Culturale Italiana. 1960-61. Cuneo 4 (1961).

118 “Agenda: Benessere, miseria e moralità II". Tempo Presente 6.1 (gennaio 1961).

119 “Agenda: Benessere, miseria e moralità III". Tempo Presente 6.2 (febbraio 1961).

120 "Le role de l'intellectuel - Silone s'explique". L'Express Parigi (9 febbraio 1961).

121 "Agenda: Benessere, miseria e moralità IV". Tempo Presente 6.3 (marzo 1961).

122 "Agenda: Con la storia in autostop". Tempo Presente 6.4-5 (aprile-maggio 1961).

123 "Agenda: Sui periodici di cultura". Tempo Presente 6.6 (giugno 1961).

124 Prefazione a Michele Pellicani. Radiografia del Comunismo. Roma: Società Editrice Democratica: 1961: 5-7.

125 "Agenda: Il mestiere di scrivere: la critica letteraria sotto processo". Tempo Presente 7.8 (agosto 1962). Rpt. "La responsabilità del mestiere di scrivere". Il Piccolo Trieste (s. a.). [Ampliato nella prefazione a Vino e pane. Milano: Mondadori, 1969.]

126 “Agenda: Angelo Tasca". Tempo Presente 7.9-10 (settembre-ottobre 1962).

127 Prefazione a Alfredo Azzaroni. Blasco, la riabilitazione di un militante rivoluzionario. Milano: Edizioni Azione Comune, 1962: 9-13. Rpt. Il 
Giorno (7 dicembre 1962); La Nazione (2 dicembre 1962); Il Resto del Carlino (3 dicembre 1962).

128 "Nichilisti e idolatri: dopo il neorealismo". Tempo Presente 7.9-10 (settembre-ottobre 1963). Rpt. "Neorealismus, Nihilismus, Idolatrie". Akzente (giugno 1967): Munich, Hanser Verlag, 1967.

129 "La terra e la gente". Abruzzo. Milano: Electa ed., 1963: 33-95.

130 “Abruzzo". Cara Italia. Milano: s. e., 1963: 77-81.

131 "Necrologi e storia". Tempo Presente 9.9-10 (settembre-ottobre 1964).

132 “A che punto è il P.C.I.?” Tempo Presente 9.11 (novembre 1964).

133 "Le luci della città". Tempo Presente 9.12 (dicembre 1964).

134 Introduzione a Michele Pellicani. La tragedia della classe operaia. Roma [?]: Edizioni Azione Comune, 1964: 11-13.

135 "Il miraggio del "partito nuovo". Tempo Presente 10.1 (gennaio 1965).

136 "Sequestri illusori". "I contadini e la neve". Tempo Presente 10.2 (febbraio 1965).

137 "Le tentazioni del potere". "La via d'uscita". Tempo Presente 10.3 (marzo 1965).

138 "Solidarietà coi poldevi?" "Statalismo e corruzione". Tempo Presente 10.5-6 (maggio-giugno 1965).

139 "Coesistenza politica e confronto delle idee". La Fiera Letteraria (27 giugno 1965). Coscienza Roma (8 settembre 1965).

140 "Martin Buber". Tempo Presente 10.7 (luglio 1965).

141 "Torpore endemico". "Vecchi otri". Tempo Presente 10.8 (agosto 1965).

142 "La virtù della sincerità". La Fiera Letteraria (19 settembre 1965).

143 "Non-violenza". "Don Milani". Tempo Presente 10.9-10 (settembre-ottobre 1965).

144 "Ecco perché mi distaccai dalla Chiesa”. La Discussione (31 ottobre 1965). Rpt. La Fiera Letteraria (7 novembre 1965).

145 "Illusioni ingiustificate". "Una profuga dell'internazionale". Tempo Presente 10.12 (dicembre 1965).

146 Prefazione a Alfredo Azzaroni. Blasco: la vie de Pietro Tresso. Parigi: Commission pour la Vérité sur les Crimes de Staline, 1965: 17-21.

147 "Intorno al processo". Tempo Presente 11.2 (febbraio 1966).

148 "I figli del partito". Tempo Presente 11.3-4 (marzo-aprile 1966). Rpt. Prefazione a Anita Galliussi. I figli del partito. S. 1.: s. e., 1966.

149 "I perseguitati persecutori". La Fiera Letteraria (5 maggio 1966).

150 "Fascismo, antifascismo e altre cose". Tempo Presente 11.6 (giugno 1966). 
151 "Begegnung mit Deutschen". Gratulatio für Joseph Gaspar Witsch zum 60 Geburstag am 17 Juli 1966. Köln: Verlag Kiepenheuer-Witsch, 1966: 269-73.

152 "Il marasma jugoslavo". Tempo Presente 11.7 (luglio 1966).

153 "Un Silone inedito". Letture (luglio 1966): 503-4.

154 "Il bandolo della matassa". "Democrazia cifrata". Tempo Presente 11.8 (agosto 1966).

155 “Questioni socialiste”. Tempo Presente 11.9-10 (settembre-ottobre 1966).

156 "Un progetto di mozione". Tempo Presente 11.11 (novembre 1966).

157 "Fine d'anno e fine di secolo". "L'esperienza di Pasolini”. Tempo Presente 11.12 (dicembre 1966).

158 "Esperienze socialiste". "Verità e tiratura". "Dov'è la stampa libera?" Tempo Presente 12.1 (gennaio 1967).

159 "Inchieste sulla stampa". Tempo Presente 12.5 (maggio 1967).

160 "False etichette". Tempo Presente 12.8 (agosto 1967).

161 "Erich un Nettie". [In un opuscolo in memoria di Nettie Sutro.] Zurich: 1967: 12-4.

162 "Trionfo e morte dei Soviet". Tempo Presente 12.11 (novembre 1967).

163 "Quel che rimane". Tempo Presente 12.12 (dicembre 1967).

164 "L'ombra dell'articolo 7, lettera al direttore". Corriere della Sera (17 febbraio 1968).

165 "Uscire dal sistema". Fede e Civiltà Parma (febbraio 1968).

166 "I fatti di Praga". Tempo Presente 13.3-4 (marzo-aprile 1968).

167 "Tecchi a Berlino". Bollettino del Sindacato Nazionale Scrittori Roma (maggio 1968).

168 "Temi per un decennio". Tempo Presente 13.11-12 (novembre-dicembre 1968).

169 "Silone-Gilas: e dopo il comunismo?" Corriere della Sera (15 maggio 1969).

170 "Da una lettera di Ignazio Silone". Critica Sociale (20 agosto-5 settembre 1969).

171 "In che senso attuale". Il Dramma (12 settembre 1969).

172 "Tradizione e aggiornamento". Panorama (16 ottobre 1969).

173 "Il mio libro incompreso. Silone: senza affanno". Corriere della Sera (12 febbraio 1970).

174 "Lenin: le idee e la realtà". Panorama (23 aprile 1970).

175 Introduzione a Folco Quilici. Abruzzo e Molise. A cura dell'Ufficio Pubbliche Relazioni della Esso Standard Italiana: novembre 1970: 9-13. 
176 "Ai piedi d'un mandorlo". Dal villaggio all'Europa. Roma: De Luca Editore, 1971.

177 Ignazio Silone zum 75 Geburtstag. Berlin: Europäische Ideen, 1973. [Contiene lettere del 1942 tra la censura militare elvetica e l'editore di Silone a proposito de Il seme sotto la neve, e corrispondenza varia inedita in italiano tra Silone ed i suoi conoscenti e collaboratori.]

University of South Australia

\section{OPERE CITATE}

D’Eramo, Luce. L'opera di Ignazio Silone: saggio critico e guida bibliografica. Mondadori: Milano, 1971.

Silone, Ignazio. Il seme solto la neve. Milano: Mondadori, 1950. Memoriale dal carcere svizzero. Roma: Lerici, 1979. Uscita di sicurezza. Milano: Longanesi, 1971. Vino e pane. Milano: Mondadori, 1955.

Signori, Elisa. "Ignazio Silone nell'esilio svizzero". Nuova Antologia (ottobre-dicembre 1979). 